\title{
HER2 Expression Discordance between Ductal Carcinoma In situ and Invasive Breast Carcinoma. How to Analyze Oncotype DX
}

\author{
Irene Ruiz¹, Freddy Fernando Durán ${ }^{1}$, Carmen Sáez¹, Julián Sanz , Adela Pelayo ${ }^{1}$, \\ Fernando Moreno ${ }^{2}$, Jorge Ruiz ${ }^{3}$, Luis Ortega ${ }^{1}$ and Alejandro Pascual ${ }^{1, *}$ \\ ${ }^{1}$ Department of Surgical Pathology; ${ }^{2}$ Department of Oncology; ${ }^{3}$ Department of Gynecology, Hospital Clínico \\ Universitario San Carlos, Madrid 28040, Spain
}

\begin{abstract}
Although histologically invasive breast carcinomas can be of no special subtype (ductal) or of special subtypes (as lobular carcinoma), based on their immunohistochemical and molecular features, they are subclassified in four different groups: luminal A, luminal B (with and without HER2 overexpression), HER2 subtype and triple negative. They vary in their gene expression signature, biological potential and clinical course. Luminal A subtype is considered to have a better prognosis and most are treated with hormone therapy alone after surgery because they express hormone receptors and show a low proliferation index. Oncotype DX (ODX) is a molecular score assay that estimates recurrence risk for early-stage hormone receptor-positive, human epidermal growth factor receptor 2 (HER2) negative breast cancer. It can predict which tumours may benefit from adjuvant chemotherapy. It has been reported that occasionally a breast carcinoma can have immunohistochemical and molecular differences between the in situ and the invasive components. We report one case that may lead us to misinterpret ODX results, where the in situ ductal component amplified HER2 gene while invasive component did not. Therefore, carefulness must be taken when evaluating ODX results, and be sure that we are evaluating the invasive component.
\end{abstract}

Keywords: HER2, DCIS, FISH, ODX.

\section{INTRODUCTION}

Breast cancer is the most frequent carcinoma in women. It is the fifth cause of death from cancer overall, but it is still the most common cause of cancer death in women in both developing and developed countries [1].

Gene expression studies have shown that there is a considerable diversity among breast carcinoma, both biologically and clinically [2]. St. Gallen classification divides breast cancer into 4 subgroups that vary in their gene expression signature, biological potential and clinical course. The classification includes: luminal $A$, luminal B (with and without HER2 overexpression), HER2 subtype and triple negative [3].

Ductal carcinoma in situ (DCIS) and invasive components of breast carcinoma usually show the same immunophenotype in both components of the same tumour. However, up to $1 \%$ of cases show different immunohistochemical profiles among these components $[4,5]$.

ODX (Genomic Health, Redwood City, CA, EE. UU) is a 21-gene breast cancer score assay that estimates recurrence risk for early-stage hormone receptorpositive, HER2-negative breast cancer [6]. It supplies

*Address correspondence to this author at the Department of Surgical Pathology, Hospital Clínico San Carlos, C/Profesor Martín Lagos s/n. Madrid 28040, Spain; Tel: +34-913303840; Fax: +34-913303504;

E-mail: alejandro.pascual@salud.madrid.org additional prognostic information and can be predictive of benefit from adjuvant chemotherapy [7]. The ODX test is currently used worldwide for routine evaluations in conjunction with Guidelines from the American Society of Clinical Oncology (ASCO), the National Comprehensive Cancer Network (NCCN), and others $[8,9]$.

We report a case where the invasive component shows luminal $A$ immunophenotype and DCIS component shows HER2 immunophenotype. We discuss the interpretation of ODX results in the surgical specimen and in the core needle biopsy.

\section{CASE REPORT}

A screening mammogram performed on an asymptomatic 49-year-old woman demonstrated a BIRADS 2 lesion in the left breast. Gynaecologicalobstetric history showed early menopause at 39 years and hormone replacement therapy for 5 years. On examination, she had small breasts with increased density in the upper outer quadrant of the left breast of $4 \mathrm{~cm}$ in length and an ipsilateral rolling adenopathy.

A core needle biopsy was performed and four cylindrical fragments were evaluated. Histological study demonstrated an invasive breast carcinoma of no special subtype (grade I of Elston-Ellis), without associated DCIS component (Figure 1A). Invasive carcinoma cells were positive for estrogens (Allred score 8) and CK19, while negative for HER2 protein 
(Figure 1B) and progesterone receptors with a low proliferation index (1\%, studied with Ki67). Therefore, it was classified as luminal A subtype.

One month after diagnosis a left mastectomy was performed with sentinel node biopsy. Macroscopic study demonstrated a mastectomy specimen that measured $12.5 \times 10 \times 3.5 \mathrm{~cm}$. Serial sections showed two indurated lesions separated by more than $2 \mathrm{~cm}$ that were located in upper-outer quadrant and in outer inter quadrants measuring $2 \times 1 \mathrm{~cm}$ and $1 \times 1 \mathrm{~cm}$ respectively. We studied 2 left axillary sentinel lymph nodes that were negative for carcinoma metastasis.

In the microscopic study, upper outer quadrant lesion was diagnosed as an invasive breast carcinoma of no special type with a peripheral rim of associated ductal carcinoma in situ component (Figure 1C). The whole tumour measured $1.8 \mathrm{~cm}$, while the central invasive component measured $1 \mathrm{~cm}$. The second tumour was a ductal carcinoma in situ grade II without necrosis and measured $1 \times 1 \mathrm{~cm}$.

ODX was requested on the surgical specimen and 15 unstained sections were evaluated. The study determined that there was amplification of the HER2 gene and recurrence score: 38, in the submitted samples. These results were disharmonious with core needle biopsy diagnosis.

Surgical specimen tissue sections were reviewed and we observed that the invasive component had

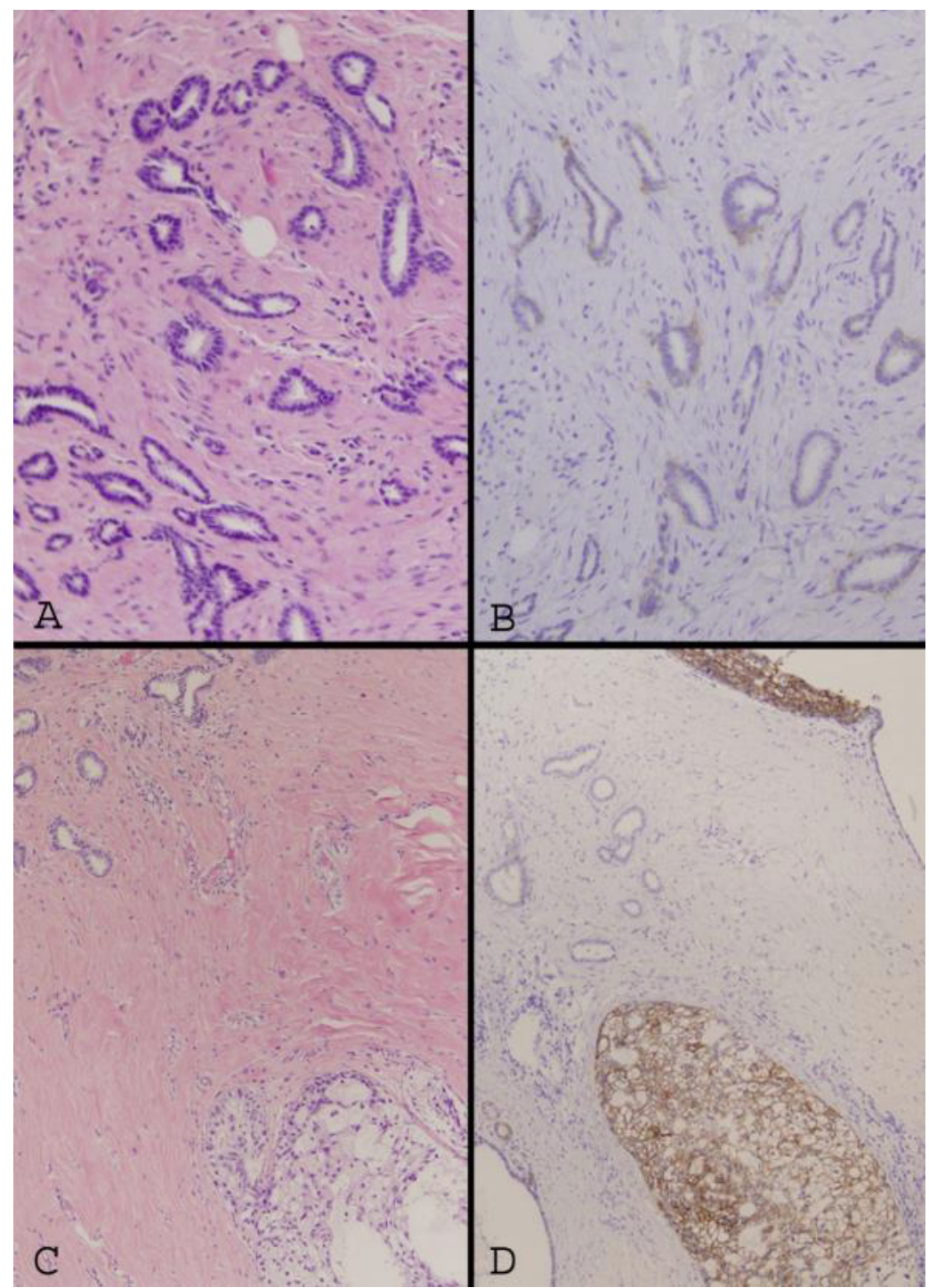

Figure 1: A. Invasive carcinoma in needle core biopsy (H \& E stain, x200 aprox). B. Invasive carcinoma showing HER2 IHC negative staining (Herceptest IHC, x200 aprox). C. In situ and invasive component of breast carcinoma (H\&E stain, x100 aprox). D. In situ and invasive carcinoma showing discordant Her2/neu protein expression. 3+ IHC membrane staining is noted within the DCIS and negative staining within the invasive component (Herceptest $\mathrm{ICH}, \mathrm{x} 100$ aprox). 
practically disappeared as a result of serial cuts, so DCIS component predominated and it was the part of the lesion that overexpressed HER2 (Figure 1D) and amplified HER2 gene by fluorescence in situ hybridization (FISH) tests (HER/cep17 ratio= 3,5 / average HER2 copy number signals/cells $=7$ ), unlike the minimum invasive component that did not overexpressed HER2 protein (Figure 2A).

Invasive component was better represented on core needle biopsy, so we repeated immunohistochemical and Fluorescence in situ hybridization (FISH) tests for HER2 protein over these sections. We obtained negative results $(\mathrm{HER} / \mathrm{cep} 17$ ratio $=1,5 /$ average HER copy number signals/cells $=1,9$ ) (Figure 2B), so we performed ODX over the core needle biopsy. The study determined that there was not amplification of the HER2 gene and recurrence score: 15.

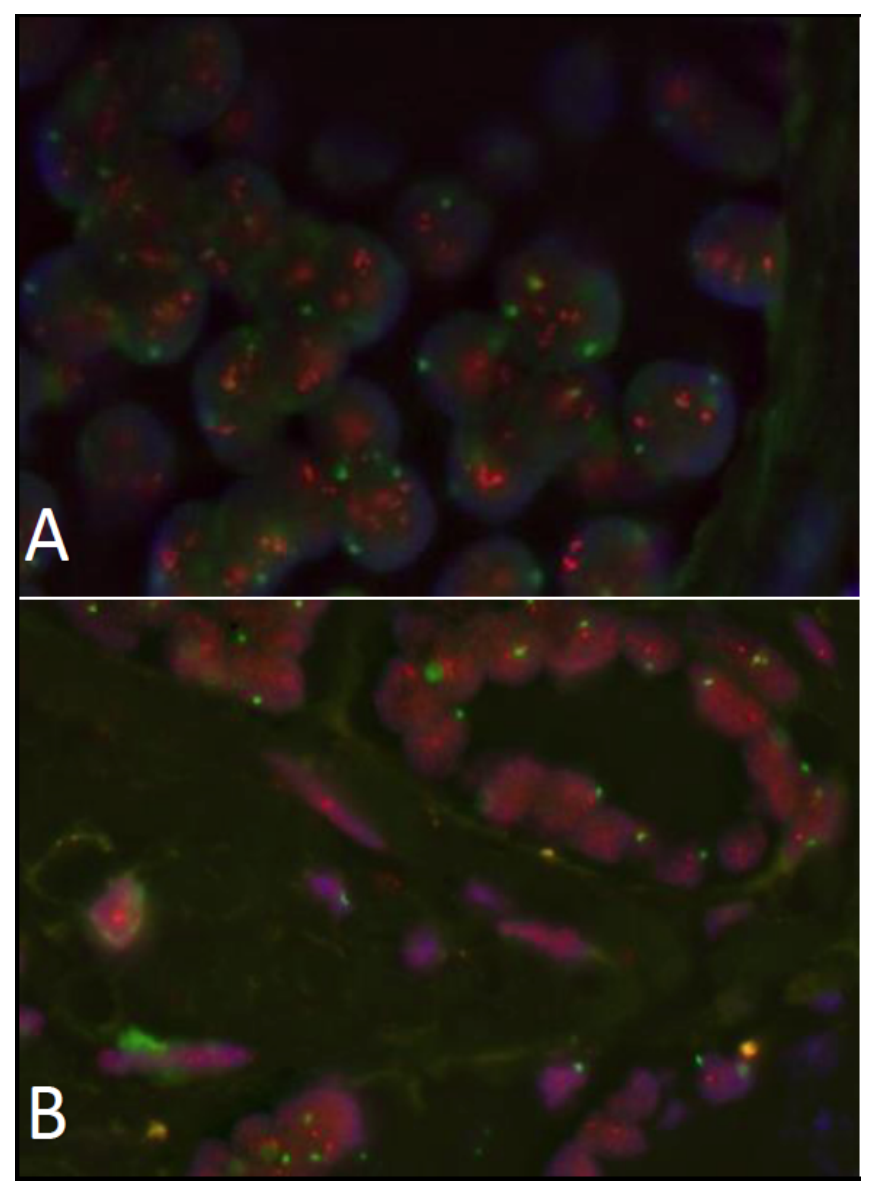

Figure 2: A. In situ component showing high HER2/neu amplification by FISH. B. Invasive ductal carcinoma showing no HER2/neu amplification.

As the infiltrating component is the one that determines prognostic course, it was concluded that it was a Luminal A carcinoma with a low recurrence rate, without benefits from adjuvant chemotherapy.
To perform the immunohistochemical staining and molecular studies, $4 \mathrm{~m} \mu$ tissue sections of the paraffin embedded material were used on slides treated with poly-L-lysine using antibodies to the following antigens: ER, PR, CK19, and Ki67 (Agilent Technologies, Inc. Santa Clara USA). Dako-OMNYS equipment was used for immunostaining. The immunodetection was carried out using the ENVISION FLEX, HIGH pH visualization system (Agilent Technologies, Inc. Santa Clara USA).

Hormone receptor evaluation was made according to ASCO/CUP 2010 recommendations [10].

Proliferation index evaluation (Ki67) was performed according to recommendations from the international Ki-67 in Breast Cancer Working group 2011 [11].

To evaluate HER2 overexpression IHC techniques were performed according to the manufacturer of the HercepTest kit (Agilent Technologies, Inc. Santa Clara USA). HER2 gene amplification was determined by FISH using a dual-colour probe, Food and Drug Administration-approved PathVysionTM HER2/neu DNA Probe Kit and Paraffin Pre-treatment Kit (Agilent Technologies, Inc. Santa Clara USA) [10].

HER2 protein overexpression evaluation (IHQ) and HER2 gene amplification (FISH), were performed according to ASCO / CUP 2013 recommendations [12].

\section{DISCUSSION}

Breast cancer is a heterogeneous disease. Most studies consider the in situ component associated with invasive carcinomas as a precursor lesion for cancer. A correlation between the molecular changes involved in the progression of the in situ component to the invasive component has been found. If left untreated, many of these in situ lesions will progress to invasive cancer. However, there are not well established histological, immunohistochemical or molecular criteria that allow us to discern which of these in situ lesions will progress to invasive carcinomas [3].

HER2 overexpression DCIS rises up to $50 \%$ of the cases, which has led to the hypothesis that HER2 amplification may be an early genetic change in the carcinogenesis of breast cancer. Local recurrence of DCIS varies depending on the studies (16-60\%) [13]. Despite its prognostic value, clinical implications of DCIS HER2 overexpression have not yet been clarified; being HER2 overexpression more frequent in high-grade DCIS lesions (50\%) than in invasive cancer samples $(20-30 \%)[14]$. 
Invasive carcinomas may have a variable in situ associated component [4]. Although immunohistochemical features are usually similar in both components there may be differences in $1 \%$ of cases. There are several reports that have described cases of invasive breast carcinoma that, unlike their accompanying in situ component, do not amplify for HER2 [5]. Therefore, there are cases, such as ours, in which HER2 status is different. Some studies such as Park et al. [4] showed four cases where in situ ductal component amplified HER2 while the accompanying invasive component did not. In this same study, a higher prevalence HER2 overexpression was identified in pure DCIS than in those with micro invasive or invasive carcinoma. These data are very important to correctly evaluate ODX results. In our case, we did not studied whether there was sufficient amount of invasive carcinoma being represented in the unstained sections of the surgical specimen submitted.

After receiving ODX report, whose results determined that there was HER2 gene amplification, new cuts of the tumour were made. In these new sections, it was observed that there was a predominance of DCIS component that overexpressed HER2, compared to the invasive component that was poorly represented (infiltrative component did not overexpressed HER2). We consider that HER2 overexpression in the in situ component was responsible for ODX assay results.

Invasive carcinoma is the lesion with prognostic value. Therefore, we decided to send new core needle biopsy tissue sections to carry out the ODX test. In the new sections invasive component was represented without the presence of a ductal in situ component. The results of this study determined that it was a low recurrence risk tumour, without HER2 amplification, and adjuvant chemotherapy was unnecessary.

\section{CONCLUSIONS}

As previous studies have shown, and our study shows, in some cases, DCIS and invasive carcinoma do not have the same immunohistochemical or molecular profile $[4,5]$. This variability can lead us to make mistakes when interpreting FISH and ODX results. Immunohistochemistry and HER2 must be performed in the surgical specimen and if they do not have the same immunohistochemical features and HER2 status it is essential to ensure that the component we analyse by ODX is the invasive and no the DCIS component, because the former is the one taken into account for therapy decisions and patients prognosis, so microdissection may be performed if necessary.

\section{REFERENCES}

[1] Ferlay J, Shin HR, Bray F, Forman D, Mathers C, Parkin DM Estimates of worldwide burden of cancer in 2008: GLOBOCAN 2008. Int J Cancer 2010; 127: 2893-917. https://doi.org/10.1002/ijc.25516

[2] Sorlie T, Perou CM, Tibshirani R, Aas T, Geisler S, Johnsen $\mathrm{H}$, et al. Gene expression patterns of breast carcinomas distinguish tumour subclasses with clinical implications. Proc Natl Acad Sci USA 2001; 98: 10869-10874.

https://doi.org/10.1073/pnas.191367098

[3] Goldrich A, Wood WC, Coates AS, Gelder RD, Thrulmann B, et al. Strategies for subtypes--dealing with the diversity of breast cancer: highlights of the St. Gallen international expert consensus on the primary therapy of early breast cancer 2011. Ann Oncol 2011; 22(8): 1736-47. https://doi.org/10.1093/annonc/mdr304

[4] Park K, Han S, Kim HJ, Kim J, Shin E. HER2 status in pure ductal carcinoma in situ and in the intraductal and invasive components of invasive ductal carcinoma determined by fluorescence in situ hybridization and immunohistochemistry. Histopathology 2006; 48(6): 702-7. https://doi.org/10.1111/j.1365-2559.2006.02403.x

[5] Aguiar FN, Mendes HN, Bacchi CE, Carvalho FM. Comparison of nuclear grade and immunohistochemical features in situ and invasive components of ductal carcinoma of breast. Rev Bras Ginecol Obstet 2013; 35(3): 97-102. https://doi.org/10.1590/S0100-72032013000300002

[6] Paik S, Shak S, Tang G, Kim C, Baker J, Cronin M, et al. A multigene assay to predict recurrence of tamoxifen-treated, node-negative breast cancer. $\mathrm{N}$ Engl J Med 2004; 351: 2817 e26.

https://doi.org/10.1056/NEJMoa041588

[7] Paik S, Tang G, Shak S, Kim C, Baker J, Kim W, et al. Gene expression and benefit of chemotherapy in women with node-negative, estrogen receptor positive breast cancer. J Clin Oncol 2006; 24: 3726e34. https://doi.org/10.1200/JCO.2005.04.7985

[8] Harris LN, Ismaila N, McShane LM, Andre F, Collyar DE, Gonzalez-Angulo AM, et al. Use of biomarkers to guide decisions on adjuvant systemic therapy for women with earlystage invasive breast cancer: American Society of Clinical Oncology clinical practice guideline. J Clin Oncol 2016; 34: 1134 e50.

https://doi.org/10.1200/JCO.2015.65.2289

[9] Henry NL, Somerfield MR, Abramson VG, Allison KH, Anders $\mathrm{CK}$, Chingos DT, et al. Role of patient and disease factors in adjuvant systemic therapy decision making for early-stage, operable breast cancer: American society of clinical oncology endorsement of cancer care Ontario guideline recommendations. J Clin Oncol 2016; 34: 2303e11. https://doi.org/10.1200/JCO.2015.65.8609

[10] Hammond ME, Hayes DF, Dowsett M, Allred DC, Hagerty $\mathrm{KL}$, Badve $\mathrm{S}$, et al. American Society of Clinical Oncology/College of American Pathologists guideline recommendations for immunohistochemical testing of estrogen and progesterone receptors in breast cancer. Arch Pathol Lab Med 2010; 134(7): e48-72.

[11] Dowsett M, Nielsen TO, A'Hern R, Bartlett J, Coombes RC, Cuzick J, et al. Assessment of $\mathrm{Ki} 67$ in breast cancer: recommendations from the International Ki67 in breast cancer working group. J Natl Cancer Inst 2011; 103(22): 1656-64.

https://doi.org/10.1093/jnci/djr393 
[12] Wolff AC, Hammond ME, Hicks DG, Dowsett M, McShane $\mathrm{LM}$, Allison $\mathrm{KH}$ et al. Recommendations for human epidermal growth factor receptor 2 testing in breast cancer: American Society of Clinical Oncology/ College of American Pathologists clinical practice guideline update. J Clin Oncol 2013; 31(31): 3997-4013.

https://doi.org/10.1200/JCO.2013.50.9984

[13] Latta EK, Tjan S, Parkes RK, O'Malley FP. The role of HER2/neu overexpression/amplification in the progression of ductal carcinoma in situ to invasive carcinoma of the breast. Mod Pathol 2002; 15(12): 1318-25.

https://doi.org/10.1097/01.MP.0000038462.62634.B1

[14] Allred DC, Clark GM, Molina R, et al. Overexpression of HER-2/neu and its relationship with other prognostic factors change during the progression of in situ to invasive breast cancer. Hum Pathol 1992; 23: 974-979.

https://doi.org/10.1016/0046-8177(92)90257-4

Received on 13-07-2019

Accepted on 16-08-2019

Published on 17-09-2019

DOI: http://dx.doi.org/10.30683/1929-2279.2019.08.01

(c) 2019 Ruiz et al.; Licensee Neoplasia Research.

This is an open access article licensed under the terms of the Creative Commons Attribution Non-Commercial License (http://creativecommons.org/licenses/by-nc/3.0/) which permits unrestricted, non-commercial use, distribution and reproduction in any medium, provided the work is properly cited. 